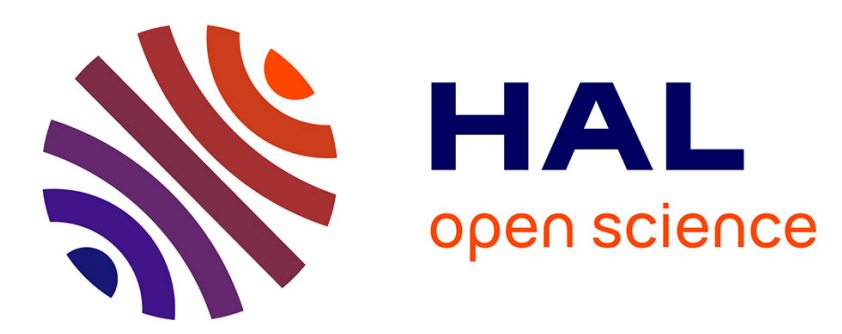

\title{
Artificial neural network for the classification of nanoparticles shape distributions
}

\author{
Y. Mansour, Y. Battie, A. En Naciri, N. Chaoui
}

\section{To cite this version:}

Y. Mansour, Y. Battie, A. En Naciri, N. Chaoui. Artificial neural network for the classification of nanoparticles shape distributions. Optics Letters, 2019, 44 (13), pp.3390-3393. 10.1364/OL.44.003390 . hal-02167692

\section{HAL Id: hal-02167692 \\ https://hal.univ-lorraine.fr/hal-02167692}

Submitted on 28 Jan 2022

HAL is a multi-disciplinary open access archive for the deposit and dissemination of scientific research documents, whether they are published or not. The documents may come from teaching and research institutions in France or abroad, or from public or private research centers.
L'archive ouverte pluridisciplinaire $\mathbf{H A L}$, est destinée au dépôt et à la diffusion de documents scientifiques de niveau recherche, publiés ou non, émanant des établissements d'enseignement et de recherche français ou étrangers, des laboratoires publics ou privés. 


\title{
Artificial Neural Network for classification of nanoparticles shape distributions
}

\author{
Y. Mansour, Y. Battie, ${ }^{*}$ A. En Naciri, AND N. ChaOUI \\ LCP-A2MC, Institut Jean Barriol, Université de Lorraine, 1 Bd Arago, 57070 Metz, France \\ *Corresponding author: yann.battie@univ-lorraine.fr
}

Received XX Month XXXX; revised XX Month, XXXX; accepted XX Month XXXX; posted XX Month XXXX (Doc. ID XXXXX); published XX Month XXXX

\begin{abstract}
A new methodology is developed to determine the shape distribution profile of gold nanoparticles (NPs) from optical spectroscopic measurements. Indeed, an artificial neural network (ANN) approach was introduced to classify $\mathrm{Au}$ nanoparticle (NP) shape distributions from their normalized absorption spectra. This ANN quantitatively analyzes the absorption spectra and provides the posterior probability to have a bimodal or unimodal shape distribution. Several colloidal suspensions were considered to investigate the robustness of the ANN approach. The comparison between ANN classification and TEM analysis was also given and discussed. We demonstrate that ANN classification is a suitable tool to inspect rapidly $\mathrm{Au}$ colloidal suspensions after their synthesis.
\end{abstract}

http://dx.doi.org/10.1364/OL.99.099999

Common elaboration processes of colloidal suspensions of $\mathrm{Au}$ nanorods (NRs) often yield a mixture of multiple nanoparticle (NP) shapes, such as nearly spherical NPs and NRs [1-3]. However, the synthesis of NRs with well-defined shape is required to investigate the shape-dependent chemical and physical properties of Au NPs. Several post synthesis separation techniques have been proposed to sort the NPs by shape [4-10]. The efficiency of the sorting techniques is mainly evaluated by transmission electron microscopy (TEM). However, since TEM is a local characterization tool, the statistical analysis of the NPs shape distributions is timeconsuming since it must be performed on a large number of NPs. Thus, the development of non-local characterization tools remains a crucial issue to control the NP shape distribution after their synthesis.

Non local technique, such as dynamic light scattering (DLS) can be used to characterized the size distribution of spherical NPs. This technique can be extended to estimate the aspect ratio of NRs from their translational and rotational relaxation rate [11-12]. However, DLS is only sensitive to the hydrodynamic size of NRs. Thus, the estimated value of aspect ratio is influenced by surfactant on the NRs surface [11-12]. Wawra et al. [13] have proposed to combine extinction spectroscopy to analytical centrifugation to estimate the shape distribution of NRs. This multidimensional approach enables to determine the absolute size of NRs. Kalomista et al. [14] have recently used mass spectrometry to detect NRs. These authors claimed that the aspect ratio of monodisperse NRs can be estimated from mass spectroscopy by analyzing the recorded signal profile. However, this technique was not applied to on complex distribution of NRs aspect ratio. Absorption spectroscopy can also be exploited to gain relevant insights on NP shape [15-17]. Indeed, Au NPs exhibit plasmon resonances whose characteristics are strongly related to the NP shape distribution [15,16]. Recent advances in optical modeling open new ways for quantitative optical characterization of Au NPs [18-21]. For this purpose, we have developed a shape distributed effective medium theory (SDEMT) to model the optical properties of ellipsoidal NPs distributed in shape [18]. We have demonstrated that the NP distribution can be determined from their absorption spectra by fitting it with the SDEMT model [18-21]. However, this technique requires prior assumptions concerning the distribution and to know, in particular, whether the distribution is unimodal or bimodal.

In this letter, our motivation is to develop a tool to distinguish colloidal suspensions with single population of NRs to those with two populations of NRs from their measured absorption spectra. This kind of tool is crucial to control colloidal suspension after their synthesis. For this purpose, an original method based on a classification concept is proposed for determining the unimodal or bimodal character of a NP shape distribution from its absorption spectra. Indeed, this method which is based on an artificial neural network (ANN), makes it possible to distinguish between colloidal suspension composed of NRs to others composed of a mixture of NRs and nearly spherical NPs. We demonstrate that the use of the absorption spectra of colloids with a neural treatment can lead to an accurate and reliable characterization of the NP shape distribution.

\section{Shape distributed effective medium theory (SDEMT)}

As we have shown previously [18], the absorption coefficient $\alpha$ of a medium composed of NRs or spherical NPs embedded in a homogeneous matrix can be described by the SDEMT model:

$$
\alpha=\frac{4 \pi}{\lambda} \operatorname{Im}\left(\sqrt{\frac{(1-f) \varepsilon_{m}+f \varepsilon_{N P} \beta}{1-f+f \beta}}\right)
$$

Due to their symmetry, NRs are assimilated to prolate NPs. Thus, the coefficient $\beta$ is given by:

$$
\begin{aligned}
\beta= & \frac{\varepsilon_{m}}{3} \int_{0}^{1 / 3} P\left(L_{1}\right)\left(\frac{1}{\varepsilon_{m}+L_{1}\left(\varepsilon_{N P}-\varepsilon_{m}\right)}+\right. \\
& \left.\frac{2}{\varepsilon_{m}+0.5\left(1-L_{1}\right)\left(\varepsilon_{N P}-\varepsilon_{m}\right)}\right) d L_{1} \cdot(2)
\end{aligned}
$$


$\mathrm{f}$ is the volume fraction of NPs. $\varepsilon_{m}$ and $\varepsilon_{N P}$ are the dielectric functions of water used as solvent and gold NPs [22], respectively. The latter is assumed to be that of the bulk material. The parameter $L_{1}$ is the depolarization factor of NPs which only depends on the NP shape. $P\left(L_{1}\right)$ is the normalized distribution of depolarization factor which is directly related to the distribution of NR shape. In the following, we assume that the unimodal distribution is composed of a single population of NRs distributed in aspect ratio:

$$
P\left(L_{1}\right)=C e^{-\frac{1}{2}\left(\frac{L_{1}-\overline{L_{1}}}{\sigma_{1}}\right)^{2}}
$$

While the bimodal distribution is composed of a mixture of NRs and almost spherical NPs:

$$
P\left(L_{1}\right)=C\left(C_{1} e^{-\frac{1}{2}\left(\frac{L_{1}-\overline{L_{1}}}{\sigma_{1}}\right)^{2}}+\left(1-C_{1}\right) e^{-\frac{1}{2}\left(\frac{L_{1}-\frac{1}{3}}{\sigma_{2}}\right)^{2}}\right)
$$

$\overline{L_{1}}$ and $\sigma_{1}$ are the mean value and standard deviation of the depolarization factor distribution of NRs, respectively. $\sigma_{2}$ is the standard deviation of depolarization factor of nearly spherical NPs. $C_{1}$ is the proportion of NRs in the bimodal distribution while $C$ is the normalization coefficient.

\section{Results and discussion}

The aim of this letter is to develop a classifier that helps to distinguish colloidal suspensions with single population of NPs to those with two populations of NPs from their measured absorption spectra. For this purpose, we implement an artificial neural network (ANN) which has multilayer perceptron architecture [23, 24] (Fig. 1). The ANN is composed of an input vector, a hidden layer and an output layer. The input vector is the normalized absorption spectra of colloids while the hidden and output layer are composed of mathematical units called neurons. The layers are linked together by connections associated with synaptic weights. As shown in Fig.1, each neuron $m$ operates as central units which applies an activation function $g$ on the weighted sum of its inputs:

$$
o_{m}=g\left(\sum_{p=0}^{P} w_{m, p} x_{p}\right) \text {. }
$$

$\mathrm{w}_{\mathrm{m}, \mathrm{p}}$ is the weigth of the connection between the input $\mathrm{x}_{\mathrm{p}}$ and the neuron $\mathrm{m}$. The activation function of hidden neurons is a sigmoid function while the softmax function is used as the activation function of output neurons. The softmax function insure the normalization of the output. This multilayer perceptron is a universal approximator which can be used to learn any relationship between its input and its output $[25,26]$. In the following, the input data are the absorption spectra of colloids. The ANN contains 18 hidden neurons and two outputs $\mathrm{s}_{1}$, $\mathrm{s}_{2}$. Note that the number of hidden neurons is obtained by using the heuristic variable selection algorithm [27]. The unimodal or bimodal character of the NP shape distribution is binary encoded by a 2-dimensional vector [ $\left.\mathrm{s}_{1}, \mathrm{~s}_{2}\right]$. Indeed, an unimodal distribution would be represented by the $[0,1]$ vector while the $[1,0]$ vector would be related to a bimodal distribution. Thus, $s_{1}$ and $s_{2}$ provide the posterior probability to have a bimodal and unimodal shape distributions, respectively. The relationship between the ANN input and output is learned during the training step. (a)

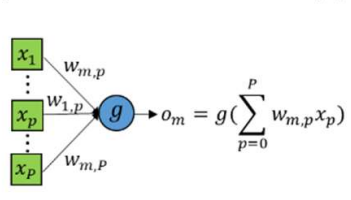



Fig. 1. (a) Calculation performed by a neural unit. $w_{m, p}$ is the synaptic weight between the input $\mathrm{x}_{\mathrm{p}}$ and the neuron $\mathrm{m}$. $\mathrm{g}$ and $\mathrm{om}$ are the activation function and the output of the neuron $m$. (b) Structure of an ANN which have a multilayer perceptron architecture. The activation functions of hidden and output neurons are a sigmoidal and a softmax functions, respectively. The input vector $\mathrm{x}_{\mathrm{p}}(\mathrm{p}=1, \ldots \mathrm{P})$ is the normalized absorption spectra of colloids. The output s1, and s2 provide the posterior probability to have bimodal and unimodal NP shape distributions, respectively.

During this step, 4000 shape distributions $P\left(L_{1}, L_{2}\right)$ of Au NPs were randomly generated by taking the parameters $\mathrm{f}, C_{n}, \overline{L_{l, n}}$ and $\sigma_{i, n}$ in the following ranges:

$$
\begin{gathered}
510^{-8} \leq f \leq 510^{-6} \\
0 \leq C_{1} \leq 1 \\
0.025 \leq \overline{L_{1}} \leq 1 / 3 \\
0.008 \leq \sigma_{1} \leq 0.1 \\
0.008 \leq \sigma_{2} \leq 0.1
\end{gathered}
$$

The absorption spectra of colloidal suspensions composed of these populations of NPs in water are then simulated from the SDEMT model. These simulations are performed for 71 wavelengths in the $400-800 \mathrm{~nm}$ spectral range. The simulated normalized absorption spectra/output vector couples are split into 3 sets: training data (1400 couples), validation data (300 couples), and test data (2000 couples). The ANN is trained with the training data by using the back propagation Levenberg-Marquardt algorithm [28]. During the training stage, the synaptic weights are adjusted to minimize the error between the desired outputs and the ANN response. To prevent overlearning and to reach the better generalization, this algorithm is stopped by using the early stopping method [25] i.e. when the error evaluated from validation data increases. Once the ANN has been trained, its performance can be estimated. The simulated normalized absorption spectra of the test data are used as new inputs of ANN. Then, the ANN outputs are compared to the expected values. The output values of $\mathrm{s}_{1}$ estimated by the ANN, shown in Fig. 2, are globally in accordance with the expected ones. The ANN performances can be quantified by the percentage of true classification. For this purpose, we define the Bayesian threshold $t_{1}$ which minimizes the number of false classification. Indeed, if $\mathrm{s}_{1}<\mathrm{t}_{1}$ the distribution is bimodal while if $\mathrm{s}_{1}>\mathrm{t}_{1}$, the distribution is unimodal. An optimal classification was obtained for $t_{1}=0.61$. $96 \%$ of the test data samples are well classified by ANN1, suggesting that a neural data processing gives an accurate characterization of the NP shape distributions. The number of false classification of unimodal distribution is twice smaller than those of bimodal distribution. Indeed, some bimodal distributions can be confused with unimodal distributions when $\mathrm{C}_{1}$ is close to 0 or close to 1 . 


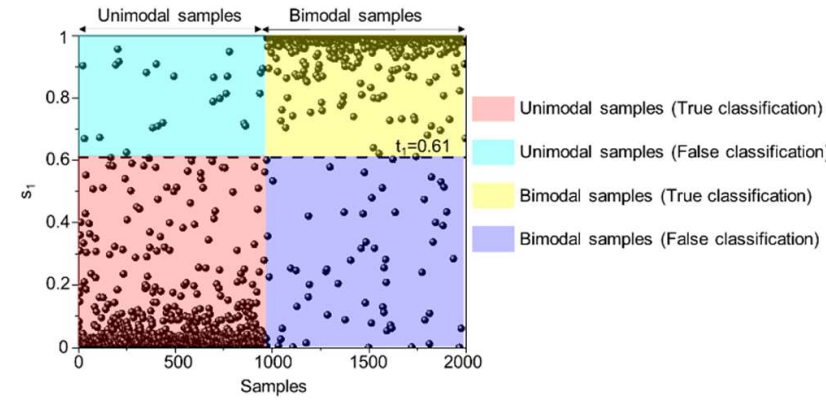

Fig. 2. $\mathrm{s}_{1}$ output of the ANN classifier calculated for 2000 test data. The Bayesian threshold $t_{1}=0.61$ which maximized the number of true classifications is depicted in dashed line.

These results (Fig.2), obtained on virtual samples, demonstrate the high level of operating performance of the ANN classifier. To validate these results, we apply the ANN to real colloidal suspensions. 12 colloidal suspensions denoted $S_{i}(i=1, \ldots, 12)$ were investigated. These suspensions were synthesized according to the seed mediated growth method [29]. The TEM images of $\mathrm{S}_{\mathrm{i}} \mathrm{Au}$ NPs, recorded with a Phillips CM200 microscope operating at $200 \mathrm{kV}$, are depicted in Figure 3(a). Au NPs are elongated in one direction and can be assimilated to prolate NPs. The NP shape distribution can be evaluated from the distribution of depolarization factor of NPs which is deduced from their aspect ratio measured by TEM [19]:

$$
L_{1}(r)=\frac{r^{2}}{1-r^{2}}\left(-1+\frac{1}{2 \sqrt{1-r^{2}}} \ln \left(\frac{1+\sqrt{1-r^{2}}}{1-\sqrt{1-r^{2}}}\right)\right),(6)
$$

where $r$ is the aspect ratio of NP defined as the ratio between the minimum and the maximum Feret diameter of NPs. The NPs shape distributions estimated from the TEM images are shown in Figure 3b.

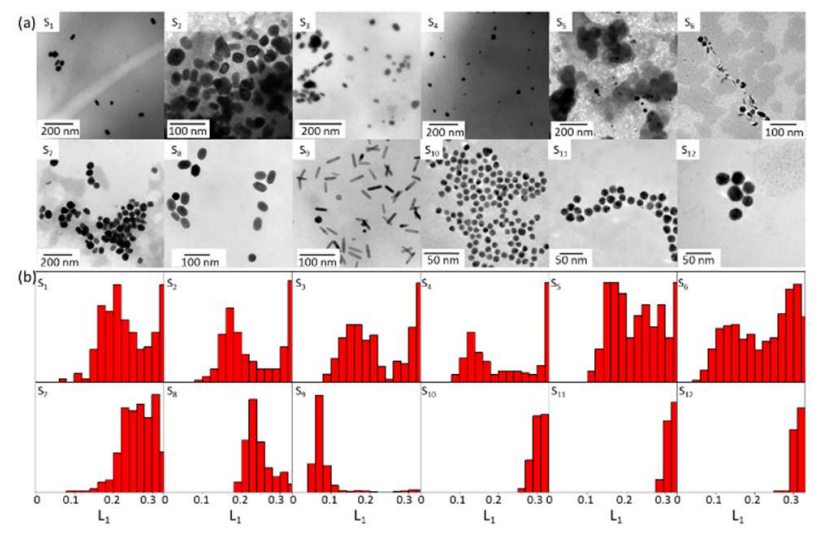

Fig. 3. (a) TEM images and (b) depolarization factor distributions of $\mathrm{Au}$ NPs contained in $\mathrm{S}_{\mathrm{i}}(\mathrm{i}=1, \ldots, 12)$ colloidal suspensions.

The $S_{1}$ to $S_{6}$ suspensions clearly exhibit a bimodal NP shape distribution. Indeed, they are composed of a mixture of nearly spherical NPs and NRs showing L1 mean value is in the 0.1-0.22 range. On the contrary, the $S_{7}$ to $S_{12}$ suspensions are composed of a unimodal NPs shape distribution. $\mathrm{S}_{7}$ shows a broad distribution of depolarization factor centered at 0.26 while $\mathrm{S}_{8}$ and $\mathrm{S}_{9}$ exhibit a narrower distribution centered at 0.23 and 0.07 , respectively. $S_{10}$, $S_{11}$, and $S_{12}$ colloidal suspensions have similar NP depolarization distributions and contain nearly spherical NPs with different sizes. As shown in TEM images, the $S_{10}, S_{11}$, and $S_{12}$ NP radii are estimated to $3 \mathrm{~nm}, 10 \mathrm{~nm}$ and $16 \mathrm{~nm}$, respectively. These suspensions are used to investigate the influence of NPs size on the ANN classification.

The normalized absorption spectra of colloidal suspensions, measured in the $400-800 \mathrm{~nm}$ spectral range with a AVANTES spectrophotometer, are shown in Fig. 4. Each suspension exhibits different spectra. This illustrates the high sensitivity of optical properties of $\mathrm{Au}$ NPs to their shape distribution. Two plasmon bands of Au NPs are clearly observed in the spectra of $S_{2}, S_{3}, S_{4}, S_{5}, S_{6}$, $\mathrm{S}_{8}$ and $\mathrm{S}_{9}$ colloidal suspensions. The first plasmon band is located in the $515 \mathrm{~nm}-550 \mathrm{~nm}$ spectral range while the second one is observed in the $600-800 \mathrm{~nm}$ range. The presence of two plasmon bands can be attributed to two plasmon modes in NRs or to bimodal NP shape distribution. On the contrary, a single plasmon band, located in the $520-600 \mathrm{~nm}$ spectral range, is observed in the spectra of $S_{1}, S_{7}, S_{10}, S_{11}$ and $S_{12}$ colloidal suspensions. $S_{1}$ and $S_{7}$ exhibits a broader plasmon band than $S_{10}, S_{11}$ and $S_{12}$.
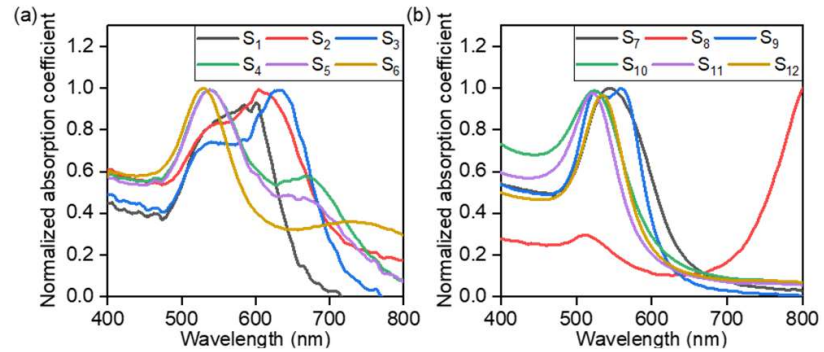

Fig. 4. Normalized absorption spectra of colloidal suspensions which contain (a) bimodal and (b) unimodal NP shape distributions.

These spectra are used as ANN inputs. The ANN classification, reported in Table I, is in accordance with TEM results excepted for suspension $\mathrm{S}_{10}$ for which the NPs mean radius is $3 \mathrm{~nm}$. As shown by several authors [30], confinement effect occurs for NP size smaller than $5 \mathrm{~nm}$. In other words, the dielectric function of $S_{10} A u$ NPs cannot be assimilated to the dielectric function of bulk. Since the training data are simulated without taking into account confinement effect, the trained ANN can only be exploited for NP sizes above $5 \mathrm{~nm}$. To overcome such problem, confinement effect should be introduced in the SDEMT model. Nevertheless, in most cases, the suspensions can be well sorted according to their distribution.

TABLE I. Comparison between TEM measurements and the ANN classification obtained for the $S_{i}(i=1, . ., 12)$ colloidal suspensions. B and $U$ denote bimodal and unimodal distributions, respectively.

\begin{tabular}{ccccccccccccc} 
& $\mathrm{S}_{1}$ & $\mathrm{~S}_{2}$ & $\mathrm{~S}_{3}$ & $\mathrm{~S}_{4}$ & $\mathrm{~S}_{5}$ & $\mathrm{~S}_{6}$ & $\mathrm{~S}_{7}$ & $\mathrm{~S}_{8}$ & $\mathrm{~S}_{9}$ & $\mathrm{~S}_{10}$ & $\mathrm{~S}_{11}$ & $\mathrm{~S}_{12}$ \\
\hline \hline & & & & & & & & & & & & \\
TEM & B & B & B & B & B & B & U & U & U & U & U & U \\
ANN & B & B & B & B & B & B & U & U & U & B & U & U
\end{tabular}


In summary, an original method based on an ANN classifier is introduced to provide qualitative information on Au NPs shape distributions from their normalized absorption spectra. This method enables the distinction between colloidal suspensions with unimodal NRs shape distribution to those with bimodal distribution. The performances of the ANN classification are evaluated on simulated and experimental data. We demonstrated that the ANN classifier is a robust and straightforward tool to characterized the NPs shape distribution provided that confinement effects are negligible. Contrary to TEM which requires a time consuming image processing and statistical analysis of NPs shape, ANN classification offers an almost instantaneous results and hence can be applied to rapidly inspect colloidal suspensions just after their synthesis.

\section{References}

1. B. D. Busbee, S. O. Obare and C. J. Murphy, Adv. Mater. 15, 414 (2003).

2. J. Pérez-Juste, L. M. Liz-Marzan, S. Carnie, D. Y. C. Chan, and P. Mulvaney, Adv. Funct. Mater. 14, 571 (2004).

3. S. E. Lohse, and C. J. Murphy, Chem. Mater. 25, 1250 (2013).

4. Z. Guo, X. Fan, L. Xu, X. Lu, C. Gu, Z. Bian, N. Gu, J. Zhang and D. Yang, Chem. Commun. 47, 4180 (2011).

5. N. R. Jana, Chem. Commun. 15, 1950 (2003).

6. V. Sharma, K. Park, and M. Srinivasarao, PNAS 106, 4981 (2009).

7. B. P. Khanal, and E. R. Zubarev, J. Am. Chem Soc. 130, 12634 (2008).

8. S. Li, Z. Chang, J. Liu, L. Bai, L. Luo, and X. Sun, Nano Res., 4, 723 (2011).

9. M. Hanauer, S. Pierrat, I. Zins, A. Lotz, and C. Sonnichsen, Nano Lett., 7, 2881 (2007).

10. G.-T. Wei, F.-K. Liu, and C. R. Chris Wang, Anal. Chem. 71, 2085 (1999).

11. J. Rodrıguez-Fernandez, J. Perez-Juste, L. M. Liz-Marzan, and P. R. Lang, J. Phys. Chem. C 111, 5020-5025 (2007).

12. M. Glidden and M. Muschol, Characterizing gold nanorods in solution using depolarized dynamic light scattering, J. Phys. Chem. C 116, 8128-8137, (2012).

13. S. E. Wawra, L. Pflug, T. Thajudeen, C. Kryschi, M.Stingl and W. Peukert, Nature Communications 9, 4898 (2018).

14. C. Noguez, J. Phys. Chem. C 111, 3806 (2007).

15. A. Resano-Garcia, Y. Battie, A. En Naciri, S. Akil, and N. Chaoui, J. Chem. Phys. 142, 134108 (2015).

16. A. Stalmashonak, G. Seifert, and H. Graener, Opt. Lett. 32, 3215-2517 (2007).

17. L. Qiu, T. A. Larson, D. Smith, E. Vitkin, M. D. Modell, B. A. Korgel, K. V. Sokolov, E. B. Hanlon, I. Itzkan, and L. T. Perelman, Appl. Phys. Lett. 93, 153106 (2008).

18. Y. Battie, A. Resano-Garcia, A. En Naciri, S. Akil, and N. Chaoui, Appl. Phys. Lett. 107, 143104 (2015).

19. Y. Battie, I. Izquierdo-Lorenzo, A. Resano-Garcia, A. En Naciri, S. Akil, P. Michel Adam, and S. Jradi, Applied Surface Science, 421, 301 (2017).

20. A. Resano-Garcia, Y. Battie, A. En Naciri, and N. Chaoui, Nanotechnology, 27, 215705 (2016).

21. E. D. Palik, Handbook of Optical Constants of Solids (Academic Press, Boston, 1998).

22. B. Yegnanarayana, Artificial neural networks (PHI Learning Pvt. Ltd., New Delhi, 2009).

23. A. Ishimaru, R. J. Marks II, L. Tsang, C. M. Lam, and D. C. Park, S. Kitamura, Opt. Lett. 15, 1221-1223 (1990).

24. C.M. Bishop, Neural Networks for Pattern Recognition, (Oxford University Press, 1996).

25. G. Cyenko, Math. Control Signal Syst. 2, 303 (1989).
26. M. Yacoub, Y. Bennani, International Journal of Neural Systems 10, 379 (2000).

27. D. Marquardt, J. Appl. Math. 11, 431 (1963).

28. B. Nikoobakht, and M. A. El-Sayed, Chem. Mater. 15, 1957 (2003).

29. Y. Battie, A. Resano-Garcia, N. Chaoui, Y. Zhang, and A. En Naciri, J. Chem. Phys. 140, 044705 (2014). 


\section{References}

1. B. D. Busbee, S. O. Obare and C. J. Murphy, An Improved Synthesis of HighAspect-Ratio Gold Nanorods, Adv. Mater. 15, 414 (2003).

2. J. Pérez-Juste, L. M. Liz-Marzan, S. Carnie, D. Y. C. Chan, and P. Mulvaney, Electric-Field-Directed Growth of Gold Nanorods in Aqueous Surfactant Solutions, Adv. Funct. Mater. 14, 571 (2004).

3. S. E. Lohse, and C. J. Murphy, The quest for shape control: A history of gold nanorod synthesis, Chem. Mater. 25, 1250 (2013).

4. Z. Guo, X. Fan, L. Xu, X. Lu, C. Gu, Z. Bian, N. Gu, J. Zhang and D. Yang, Shape separation of colloidal gold nanoparticles through salt-triggered selective precipitation, Chem. Commun. 47, 4180 (2011).

5. N. R. Jana, Nanorod shape separation using surfactant assisted selfassembly, Chem. Commun. 15, 1950 (2003).

6. V. Sharma, K. Park, and M. Srinivasarao, Shape separation of gold nanorods using centrifugation, PNAS 106, 4981 (2009).

7. B. P. Khanal, and E. R. Zubarev, Purification of High Aspect Ratio Gold Nanorods: Complete Removal of Platelets, J. Am. Chem Soc. 130, 12634 (2008).

8. S. Li, Z. Chang, J. Liu, L. Bai, L. Luo, and X. Sun, Separation of gold nanorods using density gradient ultracentrifugation, Nano Res., 4, 723 (2011).

9. M. Hanauer, S. Pierrat, I. Zins, A. Lotz, and C. Sonnichsen, , Separation of nanoparticles by gel electrophoresis according to size and shape, Nano Lett., 7, 2881 (2007).

10. G.-T. Wei, F.-K. Liu, and C. R. Chris Wang, Shape separation of nanometer gold particles by size-exclusion chromatography, Anal. Chem. 71, 2085 (1999).

11. J. Rodriguez-Fernandez, J. Perez-Juste, L. M. Liz-Marzan, and P. R. Lang, Dynamic light scattering of short Au rods with low aspect ratios, J. Phys. Chem. C 111, 5020-5025 (2007).

12. M. Glidden and M. Muschol, Characterizing gold nanorods in solution using depolarized dynamic light scattering, J. Phys. Chem. C 116, 8128-8137, (2012).

13. S. E. Wawra, L. Pflug, T. Thajudeen, C. Kryschi, M. Stingl and W. Peukert, Determination of the two-dimensional distributions of gold nanorods by multiwavelength analytical ultracentrifugation, Nature Communications 9 , 4898 (2018).

14. C. Noguez, Surface Plasmons on Metal Nanoparticles: The Influence of Shape and Physical Environment, J. Phys. Chem. C 111, 3806 (2007).

15. A. Resano-Garcia, Y. Battie, A. En Naciri, S. Akil, and N. Chaoui, Experimental and theoretical determination of the plasmonic responses and shape distribution of colloidal metallic nanoparticles, J. Chem. Phys. 142,134108 (2015)

16. A. Stalmashonak, G. Seifert, and H. Graener, Optical three-dimensional shape analysis of metallic nanoparticles after laser-induced deformation, Opt. Lett. 32, 3215-2517 (2007).

17. L. Qiu, T. A. Larson, D. Smith, E. Vitkin, M. D. Modell, B. A. Korgel, K. V. Sokolov, E. B. Hanlon, I. Itzkan, and L. T. Perelman, Observation of plasmon line broadening in single gold nanorods, Appl. Phys. Lett. 93, 153106 (2008).

18. Y. Battie, A. Resano-Garcia, A. En Naciri, S. Akil, and N. Chaoui, Determination of morphological characteristics of metallic nanoparticles based on modified Maxwell-Garnett fitting of optical responses, Appl. Phys. Lett. 107, 143104 (2015).

19. Y. Battie, I. Izquierdo-Lorenzo, A. Resano-Garcia, A. En Naciri, S. Akil, P. Michel Adam, and S. Jradi, Determination of gold nanoparticle shape from absorption spectroscopy and ellipsometry, Applied Surface Science, 421, 301 (2017).

20. A. Resano-Garcia, Y. Battie, A. En Naciri, and N. Chaoui, Interaction of a converging laser beam with a $\mathrm{Ag}$ colloidal solution during the ablation of a Ag target in water, Nanotechnology, 27, 215705 (2016).

21. E. D. Palik, Handbook of Optical Constants of Solids (Academic Press, Boston, 1998).
22. B. Yegnanarayana, Artificial neural networks (PHI Learning Pvt. Ltd., New Delhi, 2009).

23. A. Ishimaru, R. J. Marks II, L. Tsang, C. M. Lam, D. C. Park, and S. Kitamura, Particle-size distribution determination using optical sensing and neural networks, Opt. Lett. 15, 1221-1223 (1990).

24. C.M. Bishop, Neural Networks for Pattern Recognition, (Oxford University Press, 1996).

25. G. Cyenko, approximaton by superposition of sigmoidal function,Math. Control Signal Syst. 2, 303 (1989).

26. M. Yacoub, Y. Bennani, Features selection and architecture optimization in connectionist systems, International Journal of Neural Systems 10, 379 (2000).

27. D. Marquardt, An Algorithm for Least-Squares Estimation of Nonlinear Parameters, J. Appl. Math. 11, 431 (1963).

28. B. Nikoobakht, and M. A. El-Sayed, Preparation and Growth Mechanism of Gold Nanorods (NRs) Using Seed-Mediated Growth MethodChem. Mater. 15, 1957 (2003).

29. Y. Battie, A. Resano-Garcia, N. Chaoui, Y. Zhang, and A. En Naciri, Extended Maxwell-Garnett-Mie formulation applied to size dispersion of metallic nanoparticles embedded in host liquid matrix, J. Chem. Phys. 140, 044705 (2014). 